\title{
Improved axial point spread function in a two-frequency laser scanning confocal fluorescence microscope
}

\author{
Jheng-Syong Wu \\ Yung-Chin Chung \\ Jun-Jei Chien \\ Chien Chou
}




\section{Improved axial point spread function in a two-frequency laser scanning confocal fluorescence microscope}

\author{
Jheng-Syong $\mathrm{Wu}^{\mathrm{a}}{ }^{\mathrm{a}, \mathrm{b}, \dagger}$ Yung-Chin Chung, ${ }^{\mathrm{a}, \dagger}$ \\ Jun-Jei Chien, ${ }^{a}$ and Chien Chou $^{a, c, *}$ \\ ${ }^{a}$ Chang Gung University, Graduate Institute of Electro-Optical \\ Engineering, Taoyuan, Taiwan \\ ${ }^{\mathrm{b}}$ Academic Sinica, Institute of Chemistry, Nankang, Taipei, Taiwan \\ ${ }^{\circ}$ Chang Gung University, Research Center for Biomedical \\ Engineering, Taoyuan, Taiwan
}

\begin{abstract}
A two-frequency laser scanning confocal fluorescence microscope (TF-LSCFM) based on intensity modulated fluorescence signal detection was proposed. The specimen-induced spherical aberration and scattering effect were suppressed intrinsically, and high image contrast was presented due to heterodyne interference. An improved axial point spread function in a TF-LSCFM compared with a conventional laser scanning confocal fluorescence microscope was demonstrated and discussed. (0) 2018 Society of Photo-Optical Instrumentation Engineers (SPIE) [DOI: 10.1117/1.JBO.23.1.010502]
\end{abstract}

Keywords: confocal fluorescence microscopy; aberrations; optical heterodyne.

Paper 170234LRR received Apr. 21, 2017; accepted for publication Dec. 13, 2017; published online Jan. 16, 2018.

A conventional laser scanning confocal fluorescence microscope (CLSCFM) is able to reject out-of-focus photons using a microscope objective and a pinhole aperture that enable the sectioning of images for biomedical applications. ${ }^{1}$ However, the refractive-index mismatch (RIM) between the imaging object and its surroundings intrinsically induces the spherical aberration and scattering effect in specimens. This significantly degrades the sectioning image quality in a CLSCFM. ${ }^{1}$ When a biological specimen embedded in aqueous solution is probed, the specimen-induced spherical aberration (SISA) by RIM not only lowers the performance of the axial and lateral resolutions but also decreases the detected intensity at the focal point. Moreover, out-of-focus photons become easy to transmit the pinhole aperture via multiple scattering. As a result, SISA and out-of-focus scattered photons not only lower the signalto-noise ratio of the detected signal but also increase the background noise significantly. These obviously further degrade the performances of the axial and lateral point spread functions and lower the image contrast. In the CLSCFM, only pinhole aperture plays as the spatial filtering gating, which is able to reject the weak scattered photons from the defocus plane. However, the

*Address all correspondence to: Chien Chou, E-mail: cchou01@ gmail.com

${ }^{\dagger}$ Equal contribution in this research multiple scattered photons in a multiple scattering medium are not rejected properly. Those adverse effects obviously limit the quality of the sectioning images and the ability of probing biological specimens in deeper penetration depth. Hence, a way to reduce SISA and scattering effect becomes highly desired in the CLSCFM to perform better quality sectioning of images at larger penetration depths in biological specimens.

Various methods have been proposed to reduce spherical aberration in the CLSCFM: (a) adjusting the tube length of microscope objective to balance spherical aberration, (b) using an oil-immersion or water-immersion objective, (c) using beam shaping elements, ${ }^{2}(\mathrm{~d})$ associating with a linear correlation scanning microscope, ${ }^{3}$ and (e) coupling with an adaptive optical device. ${ }^{4}$ All of these methods belong to extrinsic ways of approach that compensate for the induced spherical aberration in part, while the scattering effect and SISA still exist. In our previous research, a Zeeman laser scanning confocal microscope (ZLSCM) using a two-frequency He-Ne laser as the light source, which can reduce the SISA and scattering effect simultaneously, was based on collecting the ballistic and snake linearly polarized photon-pairs (LPPP) via heterodyne interference. $^{5-8}$ The coherence imaging forming theory of the ZLSCM based on paralleled LPPP was derived. ${ }^{8}$

To focus on a confocal fluorescence microscope with less SISA and scattering effect in biomedical applications, we propose and set up a two-frequency laser scanning confocal fluorescence microscope (TF-LSCFM) based on the detection of intensity-modulated fluorescent signal excited by a two-frequency LPPP laser beam. In the TF-LSCFM, fluorophore of nanosecond in lifetime is excited by a highly spatial and temporal correlated LPPP laser beam wherein two identical and highly correlated fluorescence excitation processes in each fluorophore are produced simultaneously. An intensity-modulated fluorescence signal is emitted by heterodyne interference in optical intensity response. ${ }^{9,10}$ In terms of the polarization gating and coherence gating functioned together, the common-path propagation of polarized $p_{1}$ - and $p_{2}$-waves in the scattered LPPP laser beam automatically reduces SISA and the scattering effect in the object plane., ${ }^{7,8}$ This feature is the same as the ZLSCM of nonfluorescence imaging. Finally, the pinhole aperture integrated with an electronic filtering gating in the proposed TF-LSCFM collects the intensity-modulated fluorescence beat signal in the image plane. Thereafter, the TF-LSCFM follows the incoherence image formation theory under fluorescence signal generation. ${ }^{11}$ Finally, the TF-LSCFM becomes a hybrid image formation including the coherence image formation in the object plane and incoherence image formation in the image plane. Following the coherence image forming theory in the $\mathrm{ZLSCM}^{8}$ and the incoherence image forming theory in the CLSCFM, ${ }^{11}$ the theory of image formation of the TFLSCFM is derived. First, the intensity of the incident light onto the specimen by a point source located at $r_{0}=0$ in the source plane is expressed as

$$
\begin{aligned}
I_{0}\left(r_{1}\right)= & I_{0} \eta\left(r_{1}\right)\left[h_{1 p_{1}}\left(M_{1} r_{1}\right) h_{1 p_{2}}^{*}\left(M_{1} r_{1}\right)\right. \\
& \left.+h_{1 p_{1}}^{*}\left(M_{1} r_{1}\right) h_{1 p_{2}}\left(M_{1} r_{1}\right)\right]
\end{aligned}
$$

where the heterodyne beat signal is generated and detected at $r_{1}$ on the object plane. $I_{0}$ is the intensity of the light source, and

$1083-3668 / 2018 / \$ 25.00$ @ 2018 SPIE 
$\eta\left(r_{1}\right)$ represents the heterodyne efficiency. $r_{1}$ is the position vector in the object plane. The terms $h_{1 p_{1}}\left(M_{1} r_{1}\right)$ and $h_{1 p_{2}}\left(M_{1} r_{1}\right)$ are the 3-D complex amplitude point spread functions from the source plane to the object plane with respect to $p_{1}$ and $p_{2}$ waves, and $M_{1}$ is the demagnification matrix of the objective lens. The pupil functions in $h_{1 p_{1}}\left(M_{1} r_{1}\right)$ and $h_{1 p_{2}}\left(M_{1} r_{1}\right)$ are identical in $p_{1}$ and $p_{2}$ waves, respectively, due to their high correlation and common-path propagation in LPPP laser beam. Therefore, in the TF-LSCFM, the wavefront deformation including SISA and scattering effect induced random phase is suppressed via differential-phase detection in heterodyne beat signal. Finally, the emitted intensity-modulated fluorescence beat signal in the object plane can be expressed as

$$
\begin{aligned}
I_{1}\left(r_{1}\right)= & I_{0} \eta\left(r_{1}\right)\left[h_{1 p_{1}}\left(M_{1} r_{1}\right) h_{1 p_{2}}^{*}\left(M_{1} r_{1}\right)\right. \\
& \left.+h_{1 p_{1}}^{*}\left(M_{1} r_{1}\right) h_{1 p_{2}}\left(M_{1} r_{1}\right)\right] o_{f}\left(r_{1}\right)
\end{aligned}
$$

where $o_{f}\left(r_{1}\right)$ is the fluorescence density distribution function in the object plane. The intensity-modulated fluorescence signal is then detected by the collector lens in the image plane. As a result, the detected intensity modulated fluorescence signal at beat frequency is expressed by

$$
\begin{aligned}
I\left(r_{2}\right)= & \int_{-\infty}^{\infty} I_{0} \eta\left(r_{1}\right)\left[h_{1 p_{1}}\left(M_{1} r_{1}\right) h_{1 p_{2}}^{*}\left(M_{1} r_{1}\right)\right. \\
& \left.+h_{1 p_{1}}^{*}\left(M_{1} r_{1}\right) h_{1 p_{2}}\left(M_{1} r_{1}\right)\right] o_{f}\left(r_{1}\right)\left|h_{2}\left(r_{1}+M_{2} r_{2}\right)\right|^{2} \mathrm{~d} r_{1},
\end{aligned}
$$

where $\left|h_{2}\left(r_{1}+M_{2} r_{2}\right)\right|^{2}$ is the 3-D axial intensity point spread function via collector lens and $r_{2}$ is the position vector in the image plane. $M_{2}$ is the demagnification matrix of the collector lens. Hence, the developed image forming theory of the TFLSCFM combines the coherence imaging theory based on ballistic and snake LPPPs in the object plane with the incoherence imaging theory based on intensity modulated fluorescence signal detection in the image plane. Obviously, the TF-LSCFM reduces SISA and scattering effect by three active gatings: the polarization gating, coherence gating, and pinhole aperture gating. ${ }^{5-8}$ Meanwhile, the TF-LSCFM presents high image contrast via the electronic filtering gating, which enables it to select the intensity-modulated fluorescence beat signal where no DC bias term is involved. ${ }^{9}$

Experimentally, a sample that comprises dry-fluorescence microbeads on a glass plate was prepared and scanned in the axial direction to verify the improved axial point spread function of the TF-LSCFM in comparison with the CLSCFM. Figure 1 is the setup of the TF-LSCFM where a two-frequency He-Ne laser (Agilent, 5517A) was used. It outputted a two-frequency highly spatial and temporal correlated orthogonal linear polarized laser beam at beat frequency $1.7 \mathrm{MHz}$. A small amount of the elliptical polarization and nonorthogonality in the laser beam was produced by the linear birefringent effect in the laser cavity. ${ }^{12}$ The laser beam passes through a polarizer with an azimuth angle at $45 \mathrm{deg}$ to produce a paralleled LPPP laser beam. Hence, an intensity-modulated fluorescence signal at $1.7 \mathrm{MHz}$ was detected in the experiment. ${ }^{13}$ In the setup, a dichroic mirror separated the exciting laser beam and the fluorescent emission beam. The diameter of the pinhole aperture at $25 \mu \mathrm{m}$ was chosen to match the objective lens $O_{1}$ (Olympus, LMPLFL $100 \mathrm{X}, \mathrm{NA}=0.8, \mathrm{WD}=3.4 \mathrm{~mm}$ ) and the collector lens $\mathrm{O}_{2}$ (Lightpath Gradium lenses, $\mathrm{EFL}=60 \mathrm{~mm}, \quad f / \#=2.6$ ).

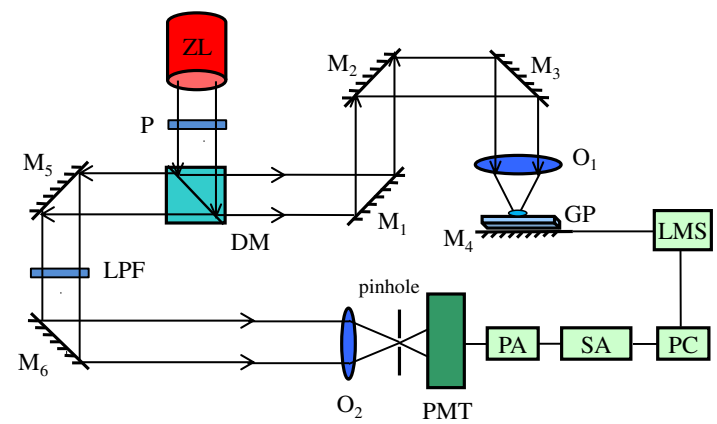

Fig. 1 Experimental setup of TF-LSCFM: ZL, Zeeman two-frequency He-Ne laser; $P$, polarizer; DM, dichroic mirror; $M_{1}-\mathrm{M}_{6}$, mirrors; $O_{1}$, objective lens; GP, glass plate; LPF, long-pass filter; $\mathrm{O}_{2}$, collector lens; PMT, photomultiplier tube; PA, preamplifier; SA, spectrum analyzer; LMS, linear motor stage.

Photomultiplier tubes (PMT) (Hamamatsu, R928) coupled with a spectrum analyzer (Agilent, N9000A) were set up for the fluorescence beat signal detection. A sample of dry-fluorescent microbeads as the imaging object was made from a fluorescent microbeads solution $(0.2 \mu \mathrm{m}$ in diameter) (Invitrogen, F8806) by dropping it onto a glass slide and then drying it. The dry-fluorescent microbeads sample was faced the objective and then axially scanned by a three-axis linear stage (Suruga Seiki, KS701-30LMS) through the focal point of the objective lens to present the axial responses of the TF-LSCFM. In order to compare the axial response of the TF-LSCFM with the CLSCFM under the same architecture, the polarizer was removed and the spectrum analyzer was replaced by a digital voltmeter at DC mode. The polarization gating and coherence gating became inactive in the CLSCFM, and total fluorescence intensity (including DC and AC signals) was measured. The normalized fluorescence axial responses (NFAR) of the TFLSCFM and CLSCFM were measured independently as shown in Fig. 2(a), where the full width half maximum (FWHM) of the fluorescence axial responses of the TFLSCFM (red line) and CLSCFM (green line) are 2.18 and $3.55 \mu \mathrm{m}$, respectively. The axial resolution of the TF-LSCFM at $1.01 \mu \mathrm{m}$ was calculated under diffraction limit without aberration. ${ }^{1}$ In Fig. 2(a), both NFARs show comparable performance on the central peaks at free of SISA when the dry-fluorescence microbeads face the objective lens in air. However, a significant reduction on background noise in the TFLSCFM compared with the CLSCFM was seen. This is because of the ability of the TF-LSCFM to suppress the multiple scattered LPPPs, which become depolarized and decorrelated in the specimen by optical heterodyne interference. Meanwhile, the weak or snake LPPPs, which become partially depolarized and decorrelated, are detected and contribute to the lower background in the fluorescence axial response in Fig. 2(a) (red line). This is in contrast to the CLSCFM where higher background [Fig. 2(a), (green line)] was contributed by multiple scattered photons produced by the clustering of the dry-fluorescence microbeads in the specimen. Furthermore, to verify the ability of SISA reduction in the TF-LSCFM, a single piece of cover glass (170 $\mu \mathrm{m}$ in thickness) was placed directly on top of the dry-fluorescence microbeads sample to introduce spherical aberration in both systems. As shown in Fig. 2(b), the FWHMs are $9.15 \mu \mathrm{m}$ in the TF-LSCFM and $21.43 \mu \mathrm{m}$ in the CLSCFM. Obviously, the TF-LSCFM shows better performance in axial response not only on FWHM in central peak but also on lower 

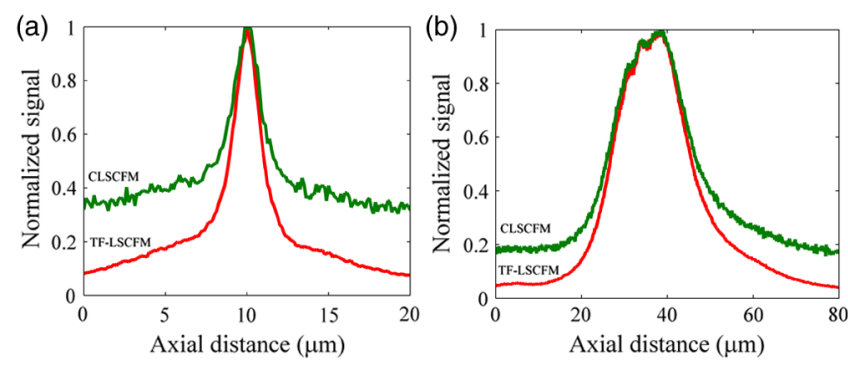

Fig. 2 NFAR measured by TF-LSCFM (red line) and CLSCFM (green line) (a) without aberration and (b) with spherical aberration by using a cover glass.

background level than that in the CLSCFM. In the TF-LSCFM, ballistic and weak scattered LPPPs in specimens are detected in terms of the polarization gating, coherence gating, and pinhole aperture gating functioned simultaneously. The highly multiple scattered LPPPs are effectively removed by polarization and coherence gatings via heterodyne interference. Experimentally, the background level cannot be removed in either the TFLSCFM or CLSCFM because the background levels in Fig. 2(a) are contributed by the scattering effect in the specimen and they are the signal-dependent background level in the fluorescence axial response. When using a perfect mirror in air as the image object, which is free of SISA and no scattering effect, there is no background level at all in the axial response measured by conventional confocal microscope in our previous study. ${ }^{8}$ This indicates that the background level in axial responses in Fig. 2(a) is indeed contributed by the weak scattered LPPPs

(a)

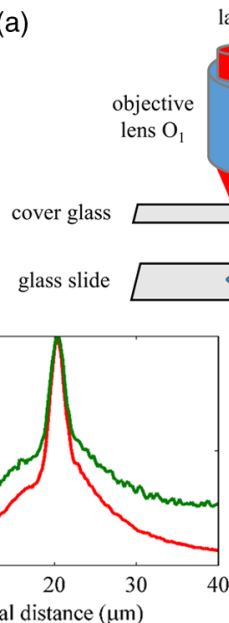

laser beam
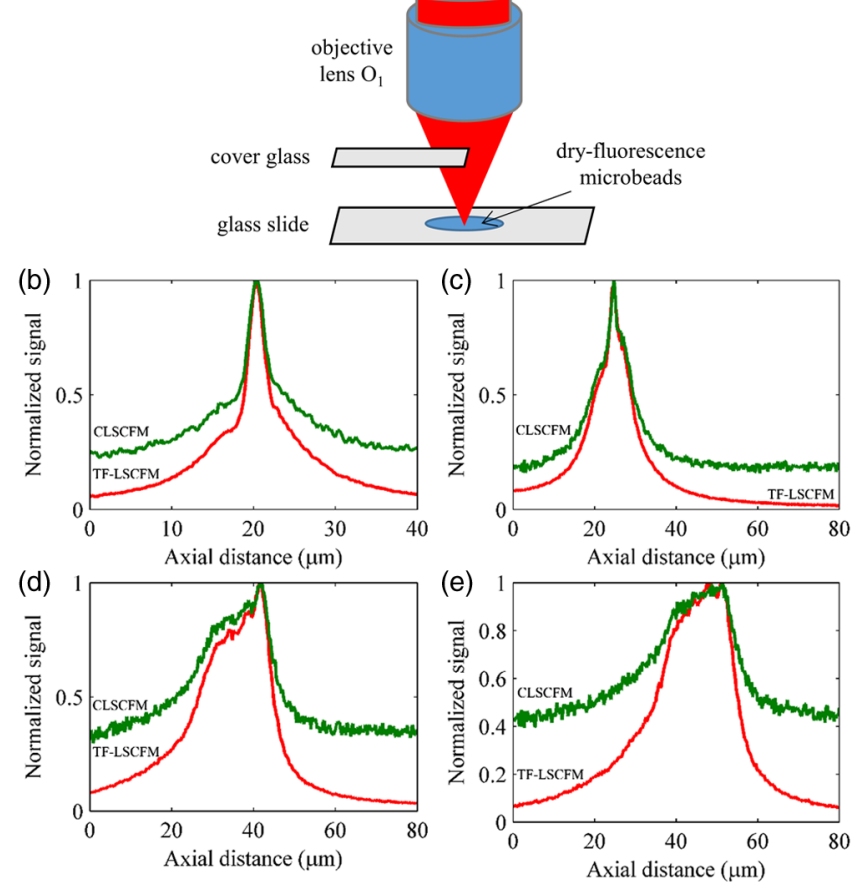

Fig. 3 (a) Setup of a piece of cover glass laterally and partially inserted into the space between objective and specimen, $(b-e)$ NFAR of TF-LSCFM (red line) and CLSCFM (green line) under different levels of SISA by cover glass covering an area at (b) $25 \%$, (c) $50 \%$, (d) $75 \%$, and (e) $100 \%$ of full aperture of microscopic objective as shown in (a). in the TF-LSCFM and by the multiple scattered photons in the CLSCFM accordingly.

To further verify the ability of the TF-LSCFM on reducing SISA, an arrangement of introducing SISA at different levels was implemented by partially covering the image object with a single piece of cover glass (170 $\mu \mathrm{m}$ in thickness). The experimental setup at different levels of SISA is shown in Fig. 3(a), where a single piece of cover glass was laterally and partially inserted into the space at the mid position between the objective $O_{1}$ and the dry-fluorescence microbeads sample. A lower SISA is anticipated than it is under the setup of placing a single piece of cover glass directly on top of the dry-fluorescence microbeads. In the experiment, the cover glass of $<170 \mu \mathrm{m}$ in thickness was not commercially available either. Because SISA is NA dependent, ${ }^{14}$ the greater percentage of the covered area in full aperture of the objective by a cover glass, the more SISA is introduced in the confocal microscope. Qualitatively, different degrees of distorted wavefront, which are proportional to the percentage of the inserted area of the cover glass, are normalized by the area of full aperture of the objective at the same location that was set up. As a result, a series of measured NFAR of the TF-LSCFM and CLSCFM under different levels of SISA is shown in Figs. 3(b)-3(e). Improvements of axial responses under different percentages of the cover area are clearly seen at (b) $25 \%$, (c) $50 \%$, (d) $75 \%$, and (e) $100 \%$ of the full aperture of objective. Because SISA not only decreases the intensity at focus but also broadens the width of the central peak in the axial responses, Figs. 3(b) to 3(e) clearly show the broadening of the FWHM in axial responses under different levels of SISA. Figure 4(a) shows the performance on the FWHM of the axial responses of the TF-LSCFM (red dots) and CLSCFM (green triangles). In addition, Fig. 4(b) shows the comparison of the FWHM between the TF-LSCFM (red dots) and the commercially available confocal microscope (Olympus FV1000, Japan) (blue crosses) with the identical microscope objective. Then, an improved fluorescence axial response was demonstrated in the TF-LSCFM under different degrees of SISA induced by a single piece of cover glass.

In this study, the TF-LSCFM is focused on the ballistic and snake LPPPs in the object plane where the intensity-modulated fluorescence signal is emitted through polarization gating and spatial coherence gating under optical heterodyne and intensity response. It belongs to the coherence imaging forming theory ${ }^{8}$ in the object plane. However, it becomes incoherence imaging ${ }^{11}$ by the fluorescence signal detection in the image plane. Hence, the abilities of suppression of SISA and multiple scattering effect in the specimen are applicable in the TF-LSCFM. This results from the polarization and coherence gatings in terms of differentialphase detection via the heterodyne interference in optical intensity response. Moreover, pinhole aperture enables the rejection of LPPPs from neighbor object planes to result in the sectioning image. Finally, the electronic filtering gating selects the intensity-modulated fluorescence signal that further enhances the image contrast in the sectioning image. In addition, the weak scattering effect in the specimen introduces random phase both in $p_{1}$ and $p_{2}$ waves simultaneously and equally. Thus, the scattering effect can be suppressed effectively as well by means of the differential-phase detection in the TF-LSCFM. A clearer sectioning image of a "starfish" in Fig. 5(a) by the TF-LSCFM versus the blur image in Fig. 5(b) by the CLSCFM verifies the ability of TF-LSCFM, wherein the "starfish" was made by dryfluorescence microbeads covered by a cover glass. Qualitatively, 


\section{JBO Letters}
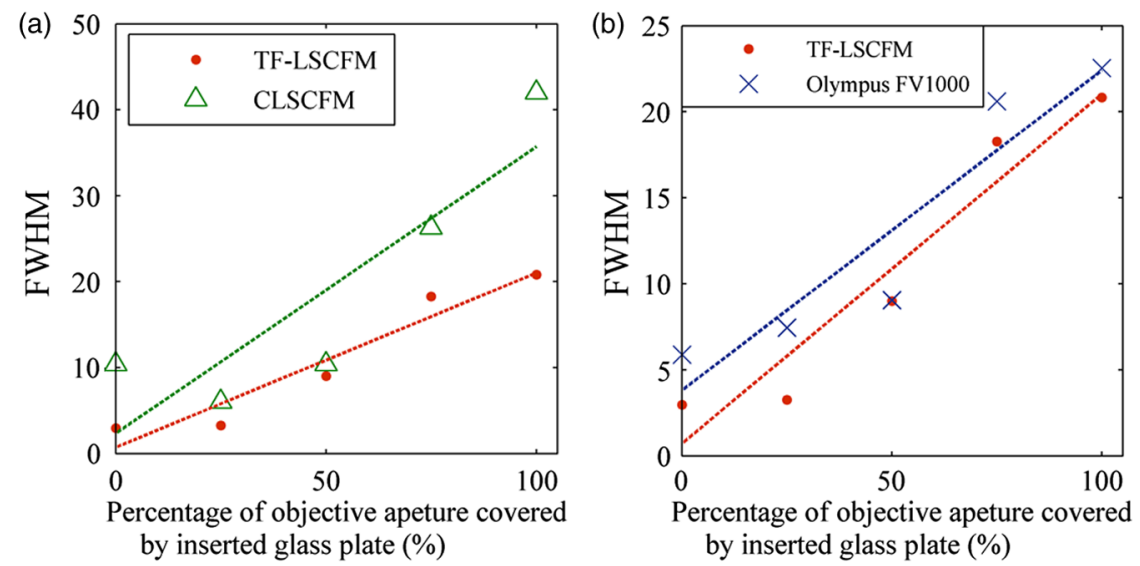

Fig. 4 FWHM of NFAR under different levels of SISA by (a) TF-LSCFM (red dots) and CLSCFM (green triangles) and (b) TF-LSCFM (red dots) and Olympus FV1000 (blue crosses). The dotted lines are the best fitting curves in a different setup of the confocal microscope.
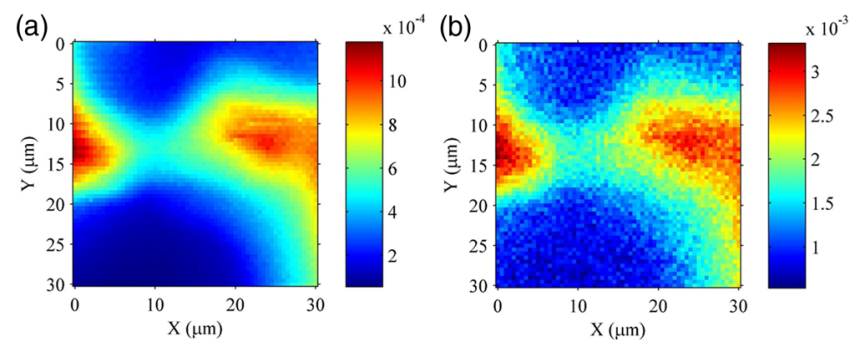

Fig. 5 Sectioning images of "starfish" sample using (a) TF-LSCFM and (b) CLSCFM.

higher image contrast, less scattering effect, and improved axial response in the TF-LSCFM than that in the CLSCFM are demonstrated and discussed. These are featured by the commonpath propagation of LPPPs and the heterodyne interference. According to the developed image forming theory of the TFLSCFM, the improvement on lateral resolution in the sectioning image is anticipated as well due to the ability of reducing SISA in the TF-LSCFM. Moreover, a nonpolarized TF-LSCFM is applicable to the integration of a single frequency laser beam with a frequency shifter for the nonpolarized two-frequency laser beam in this setup.

\section{Disclosures}

No conflicts of interest, financial or otherwise, are declared by the authors.

\section{Acknowledgment}

Olympus FV1000 supported by Professor Y. Hwu of the Institute of Physics in Academic Sinica of Taiwan is greatly acknowledged.

\section{References}

1. J. B. Pawley, Handbook of Biological Confocal Microscopy, Plenum Press, New York (1995).

2. I. Escobar et al., "Reduction of the spherical aberration effect in highnumerical-aperture optical scanning instruments," J. Opt. Soc. Am. A 23, 3150-3155 (2006).

3. M. Kempe and W. Rudolph, "Scanning microscopy through thick layers based on linear correlation," Opt. Lett. 19, 1919-1921 (1994).

4. M. J. Booth et al., "Adaptive aberration correction in a confocal microscope," Proc. Natl. Acad. Sci. U.S.A. 99, 5788-5792 (2002).

5. C. Chou et al., "Polarized optical coherence imaging in turbid media by use of a Zeeman laser," Opt. Lett. 25, 1517-1519 (2000).

6. L. C. Peng et al., "Zeeman laser-scanning confocal microscopy in turbid media," Opt. Lett. 26, 349-351 (2001).

7. H. F. Chang et al., "Angular distribution of polarized photon-pairs in a scattering medium with a Zeeman laser scanning confocal microscope," J. Microsc. 223, 26-32 (2006).

8. J. S. Wu et al., "Zeeman laser scanning confocal microscope and its ability on reduction of specimen-induced spherical aberration," Opt. Express 18, 13136-13150 (2010).

9. Y. H. Chan et al., "Optical properties of diffused photon pair density wave in a multiple scattering medium," Appl. Opt. 44, 1416-1425 (2005).

10. J. S. Wu, L. P. Yu, and C. Chou, "Measurement of the surface effect of a small scattering object in a highly scattering medium by use of diffuse photon-pairs density wave," J. Biomed. Opt. 21, 060504 (2016).

11. M. Gu, Principles of Three-Dimensional Imaging in Confocal Microscopes, World Scientific, Singapore (1996).

12. Y. Xie and Y. Z. Wu, "Elliptical polarization and nonorthogonality of stabilized Zeeman laser output," Appl. Opt. 28, 2043-2046 (1989).

13. S. Pant et al., "Line-scan focal modulation microscopy," J. Biomed. Opt. 22, 050502 (2017).

14. D. Malacara, Optical Shop Testing, p. 489, John Wiley and Sons, New York (1978). 